\title{
Outcomes of Cytoreductive Surgery (CRS) with Hyperthermic Intraperitoneal Chemotherapy (HIPEC) in Patients Older Than 70 Years; Survival Benefit at Considerable Morbidity and Mortality
}

\author{
Konstantinos I. Votanopoulos, MD, PhD ${ }^{1}$, Naeem A. Newman, MD ${ }^{1}$, Greg Russell, MS ${ }^{2}$, \\ Chukwuemeka Ihemelandu, MD ${ }^{1}$, Perry Shen, MD ${ }^{1}$, John H. Stewart, MD ${ }^{1}$, and Edward A. \\ Levine, MD ${ }^{1}$ \\ Konstantinos I. Votanopoulos: kvotanop@wakehealth.edu \\ ${ }^{1}$ Surgical Oncology Service, Department of General Surgery, Wake Forest University, Winston- \\ Salem, NC \\ ${ }^{2}$ Department of Biostatistics, Wake Forest University, Winston-Salem, NC
}

\section{Abstract}

\begin{abstract}
Background-Cytoreductive surgery (CRS)/Hyperthermic Intraperitoneal Chemotherapy (HIPEC) is associated with prolonged survival in selected patients with peritoneal surface disease. Yet, for elderly patients (older than 70 years of age) CRS/HIPEC is controversial, due to associated morbidity.
\end{abstract}

\begin{abstract}
Methods-A retrospective analysis of a prospective database of 950 procedures was performed. Type of malignancy, demographics, performance and resection status, hospitalization, morbidity, mortality, and survival were reviewed.

Results-A total of 81 patients (median age 73, range 70-87) underwent CRS/HIPEC between 1991 and 2011. Median follow-up was 48.1 months. Complete cytoreduction was achieved in 44 $\%$. Median survival was 31.8 months for appendiceal cancer, 41.5 for mesothelioma, 54.0 for ovarian cancer, 13.2 for colon cancer, and 7.6 for gastric cancer. The 30-day mortality was 13.6 $\%$. The combined grade III and IV morbidity was $38 \%$. Median ICU and hospital stay for uncomplicated patients was 1 and 8 days, respectively. The 3 -month mortality was $27.4 \%$. There were no deaths in the octogenarian group. In step-wise multivariate analysis, type of primary $(p=$ $0.03)$, albumin $(p=0.02)$, and R status $(p=0.007)$ were predictive of survival only in the absence of complications. Splitting the data at the midpoint of surgical experience, there was a drop in 1and 3-month mortality over time to 9.5 and $19.3 \%$, respectively, while the median survival increased from $11.2(N=39)$ to 46.9 months $(N=42)$.
\end{abstract}

Conclusions-HIPEC in the elderly is associated with a steep learning curve and considerable morbidity and mortality. However, age alone is not a contraindication for the procedure. Institutional experience and stringent patient selection are key factors for prolonged survival.

\begin{abstract}
The population of the United States is both growing and aging, and the number of elderly patients with cancer requiring surgical intervention is expected to rise markedly in the next decade. ${ }^{1}$ According to the Social Security Actuarial Survival Tables, the life expectancy of a person reaching the age of 70 is 14 years; furthermore an 80 -year-old person has an approximate life expectancy of 8 years. The life expectancy of elderly patients is often underestimated, and therefore these patients are frequently undertreated.
\end{abstract}

(C) Society of Surgical Oncology 2013

Presented at the 65th Annual Meeting of the Society of Surgical Oncology, Orlando, FL, March 2012. 
Over the last 2 decades, cytoreductive surgery (CRS) combined with intraperitoneal hyperthermic chemotherapy (HIPEC) has been established as a promising treatment strategy for selected patients with peritoneal surface disease (PSD), predominantly for appendiceal, colorectal, and peritoneal mesothelioma primaries. ${ }^{2-8}$ At the same time, the proportion of older people with PSD has increased following in parallel the increase of the elderly population. Outcomes on patients older than 70 years, treated with CRS/HIPEC, do not exist in the literature.

The primary aim of the present study is to assess the outcomes of CRS/HIPEC procedures in patients older than 70 and to demonstrate how advanced age impacts morbidity and mortality. The secondary aim is to identify the population of elderly patients that will obtain a survival benefit from the procedure.

\section{METHODS}

This is a retrospective analysis of a prospectively maintained database of $950 \mathrm{CRS} / \mathrm{HIPEC}$ procedures. An IRB approval was obtained. Patients who were included underwent CRS/ HIPEC after their 70th birthday. Data relevant to the analysis included demographics, Eastern Cooperative Oncology Group (ECOG) performance status, R status of resection, type of malignancy, comorbidities, morbidity, mortality, and survival. Eligibility criteria for CRS/HIPEC were ECOG $\leq 2$, pathologic diagnosis of PSD, complete recovery from prior systemic chemotherapy or radiation treatments, resectable or resected primary, debulkable peritoneal disease, and no extra-abdominal disease. Age, per se, was not a selection criterion for the procedure, but was considered by the evaluating surgeon. Peripheral liver disease, if easily resectable, was not a contraindication. All patients preoperatively had a complete history and physical, tumor markers, and CT of the chest, abdomen, and pelvis. The procedure was conducted as previously described by our group. ${ }^{9}$ Surgical morbidity and mortality were recorded according to Clavien and Dindo classification system. ${ }^{10} \mathrm{R} 0$ and R1 resections were grouped together as complete cytoreductions. Cytoreductions with residual macroscopic disease were characterized as $\mathrm{R} 2$ and subdivided based on the size of residual disease (R2a $\leq 5 \mathrm{~mm}, \mathrm{R} 2 \mathrm{~b} \leq 2 \mathrm{~cm}, \mathrm{R} 2 \mathrm{c}>2 \mathrm{~cm}$ ).

Descriptive statistics including means and standard deviations for continuous data and frequencies and percentages for categorical data were calculated. Overall survival (OS) was calculated from the date of CRS/HIPEC to the last known date of follow-up or the date of death. Estimates of survival were calculated by using the Kaplan-Meier (product-limit) method; analysis with Cox proportional hazards was performed on all pertinent clinicopathologic variables to determine each variable's association with survival. Group comparisons of OS were performed using the approximate Chi square statistic for the logrank test. Additionally, the Cox proportional hazards regression model was used in a stepwise fashion to perform a multivariate analysis of clinical and pathologic factors to determine an overall model of independent predictors of OS. Statistical significance was defined as a $p$ value $<0.05$.

\section{RESULTS}

A total of 81 elderly patients (median age, 73 years; range, 70-87 years) underwent CRS/ HIPEC between 1991 and 2011. Median follow-up was 48.1 months. Of the 81 patients, 59 $(73 \%)$ were between the ages of 70 and 74 years, $16(20 \%)$ were between the ages of 75 and 79 years, and $7(9 \%)$ were between the ages of 80 and 87 years. The distribution of primaries was 32 appendiceal cancers, 20 colon cancers, 10 mesotheliomas, 8 ovarian cancers, 6 gastric cancers, and 5 other ( 2 sarcomas, 1 rectal, 1 liver, and 1 unknown) (Table $1)$. 
A colostomy was required for 6 patients ( $7 \%$ ), and 9 patients (11\%) required an ileostomy. A total of 63 patients $(78 \%$ ) were ECOG 0/1, while 14 (17\%) were ECOG 2. R0 or R1 resection was obtained in 36 patients (44\%). R2a resection was obtained in 24 patients (30 $\%)$ (Table 1).

Median ICU and hospital stay for patients without complications were 1 and 8 days, respectively. The average hospitalization was $9.5 \pm 4.6$ days. Patients with complications had an average hospitalization of $23.4 \pm 22.9$ days with a median of 4 ICU and 15 floor days. Minor morbidity (grade I and II) was $28 \%$ (23 patients), while major morbidity (grade III and IV) was $38 \%$ (31 patients). The 30-day mortality for the entire cohort was $13.6 \%$. The 3-month mortality was $27.4 \%$. There were no deaths in the octogenarian group.

\section{Survival and Prognostic Factors in Univariate and Multivariate Analysis (Table 2)}

To evaluate the effect of institutional experience on survival, we divided the elderly cohort into 2 groups of similar volume of cases; the initial group included 1991 to 2003, with the latter being after 2004. The 2 survival curves were significantly different $(p=0.009)$. There was a reduction in 1- and 3-month mortality over time from 17.9 and $35.9 \%$ to 9.5 and 19.3 $\%$, respectively, while the median survival increased from 11.2 months $(n=39)$ to 46.9 months $(n=42)$ (Fig. 1). In addition, the probability of death was reduced by $7 \%$ for each annual increment in the institution's experience $(p=0.006)$.

When we looked at the impact of complications on survival we found that patients without complications $(n=27)$ had 1-year survival of $81 \%( \pm 8 \%)$ and 3-year survival of $59 \%( \pm 10$ $\%)$, while the median survival was 39 months. Patients with postoperative complications ( $n$ $=54)$ had 1-year survival of $53 \%( \pm 7 \%)$ and 3-year survival of $25 \%( \pm 7 \%)$, while the median survival was 13 months. Stepwise multivariate models were created with and without complications as a variable. The presence of any complication was highly associated with survival in univariate analysis $(p=0.004)$ (Fig. 2). For patients that we followed for more than 2 years, the median OS was 64.9 months. A total of 25 patients (30.9\%) lived 2 years.

The median survival stratified by primary, was 31.8 months for appendiceal cancer, 41.5 months for mesothelioma, 54 months for ovarian cancer, 13.2 months for colon cancer, and 7.6 months for gastric cancer. Type of primary was a significant predictor of survival in univariate analysis. In multivariate analysis $(p=0.03)$, the type of primary was significant only if the stepwise modeling was done without including complications (Fig. 3).

The average albumin level of patients with complications was $3.7 \mathrm{~g} / \mathrm{dL}$. The average albumin level of those patients who suffered mortality was $3.2 \mathrm{~g} / \mathrm{dL}$. Albumin was a significant predictor of survival in univariate analysis and in multivariate analysis without complications in the stepwise model $(p=0.02)$. The risk of death was reduced by $40 \%$ for each additional gram of albumin.

Preoperative hemoglobin level was significant in univariate analysis $(p=0.02)$. Patients presenting with lower hemoglobin levels had worse prognosis, with hemoglobin probably acting as a surrogate to advanced stage of disease, poor nutritional status, and often prior chemotherapy. Age and ECOG functional status were not significant in both univariate and multivariate analysis (Table 2).

In univariate analysis, the completeness of cytoreduction was significant only for patients who had no complications $(p=0.04)$. In multivariate analysis, reduced survival was directly related to the presence of postoperative complications. The completeness of cytoreduction was significant $(p=0.007)$ only for the model that did not include complications as a 
covariate. In the model without complications the type of primary $(p=0.03)$, albumin levels $(p=0.02)$, and completion of cytoreduction $(p=0.007)$ were indicative of improved survival. With complications in the stepwise model, only the institutional experience ( $p=$ $0.001)$ and the absence of complication itself $(p=0.001)$ remained and were significantly associated with survival (Table 2).

We also examined if complication rates and the associated poor survival were a function of disease burden. On multivariate analysis, the number of organs resected was significantly higher in the group with complications ( $3.3 \pm 1.7$ vs $2.1 \pm 1.3$, medians of 2 and 3 , respectively) $(p=0.003)$, meaning a higher volume of disease requires a more extensive cytoreduction and an increase in complication rate.

In addition, we included comorbidities such as diabetes, heart disease (coronary artery disease, prior MI, congestive heart failure), and lung disease (COPD, asthma) in the analysis. Using a logistic regression model, the effect of 1 comorbid condition leads to a more than doubling of the odds of having a complication with an odds ratio of 2.19 (95\% CI $1.06-4.53, p=0.035$ ). Using a multivariate analysis, we were able to determine that 19 of 50 (38\%) of smokers had complications vs 2 of 24 nonsmokers $(8 \%)(p=0.012)$.

We also compared data on patients $<70$ years versus their elderly counterparts to determine the difference in mortality and complication rates. The rate of mortality is higher in the $>70$ group than in the $<70$ group at 1 and 3 months $(p<0.0001)$. The 1 -month mortality is $3.9 \%$ in the $<70$ group versus $13.6 \%$ in the $>70$ group. The 3 -month mortality was $10.2 \%$ in the $<70$ group and $27.4 \%$ in the $>70$ group. Minor morbidity (Clavien I/II) is not significantly different between the 2 groups, with $28 \%$ of elderly patients with a minor morbidity vs 39 $\%$ in the younger patients $(p=0.07)$. There was a significant difference in major morbidity (Clavien III/IV), with $23 \%$ of the younger patients having a major morbidity versus $38 \%$ in the elderly $(p=0.002)$.

\section{DISCUSSION}

Elderly patients have been either under-represented or excluded in most prospective randomized clinical trials. ${ }^{11}$ Therefore, it is unknown whether treatment guidelines for the elderly should differ from those applied to younger patients. For the purposes of this manuscript, elderly was defined as older than 70 years, given that with the current standards of living, the age of 65 cannot always be considered the entrance into the elderly life. In addition, because of medical care advances, 1-month reports of morbidity and mortality do not capture the physiologic impact of a complex surgical procedure. Therefore, this manuscript is focused predominantly on 3-month surgical outcomes.

It has been previously shown that elderly patients are at increased risk for postoperative morbidity. ${ }^{12}$ Given the extent of surgery necessary to obtain optimal cytoreduction, the morbidity and mortality of CRS/HIPEC are not insignificant. Current morbidity rates experienced by centers performing CRS/HIPEC in patients of all ages, range from 27 to 56 $\% .{ }^{13,14}$ Our CRS/HIPEC data in this elderly subset revealed a combined grade III and IV morbidity rate of $38 \%$.

The 3-month mortality of $27 \%$ for the entire cohort is definitely increased compared with younger subjects and should be regarded only as a historic value for 1 major reason. It describes the historic evolution of a surgical procedure that was largely unknown 20 years ago and therefore includes patients that currently would not be considered candidates for CRS/HIPEC. At the start of our institutional experience 20 years ago, the key element driving the decision-making process was that patients with PSD had 4-6 months of 
anticipated survival. Thus we include in this analysis patients taken for the procedure prior to our understanding of the prognostic features outlined previously. Over the 20 years our outcomes have improved predominantly as a result of better patient selection. Patients must be medically fit with ECOG status $\underline{s}$. Elderly patients with high-volume disease, notably a PCI > 20, will not be taken to the operating room with the exception of low-grade appendiceal patients who have a favorable biological behavior. Parenchymal hepatic metastases must be resectable without having to undergo a complex operation. We also select patients based on response to neoadjuvant chemotherapy. If response to the neoadjuvant therapy is good, with documented tumor reduction, the patient may be considered for CRS/HIPEC depending on the primary and distribution of disease. There have been no randomized trials to validate these practice changes as of yet.

In addition, the operation itself is challenging with a long learning curve. ${ }^{15}$ When we looked at the effect on survival of prior CRS/HIPEC accumulated surgical experience, we calculated a $7 \%$ annual risk reduction for a patient undergoing a cytoreduction to die from the operation. This $7 \%$ annual risk reduction was stable over the entire period of 20 years and explains why the last 42 patients, when analyzed separately, demonstrated a drop in 1and 3-month mortality from 17.9 and 35.9 to 9.5 and $19.3 \%$, respectively. As volume increased, there was an increase in survival from 11 to 47 months because surgical experience and selection criteria were gradually developed and applied. The aforementioned numbers have to be taken into consideration by novice centers that are planning to develop a peritoneal surface malignancy program.

However, there is still no doubt that 9.5 and $19.3 \% 1$ - and 3-month mortality is daunting and significantly higher than what has been previously reported. ${ }^{16}$

Therefore, there are 2 questions that need to be answered: (1) Should we even consider performing a cytoreduction in a patient older than 70 years? (2) Who is the elderly patient that will benefit from a CRS/HIPEC procedure? Considering the almost 4 years (47 months) median survival obtained from the procedure, it would be difficult to argue that CRS should not be offered as a therapeutic option to selected elderly patients with PSD.

To answer the question of who is the elderly patient that will obtain a survival benefit from the procedure, we examined our data through a number of different lenses. The predictive role of ECOG performance status has been extensively studied. ${ }^{7,14}$ Younger patients with performance status of ECOG 0 or 1 have superior survival over ECOG 2 patients. In elderly patients, ECOG has less prognostic significance, possibly because it functions as an expression of both physiologic aging and volume of disease. ${ }^{17}$ On the contrary, in young patients, performance status is prognostic of survival because it records functional loss as an indirect measure of volume of disease. As an alternative to ECOG, frailty has been identified as a marker of physiologic reserves. ${ }^{18}$ A prospective analysis is needed to validate whether frailty can predict which elderly patients will do well following CRS/HIPEC. We do not offer CRS/HIPEC to patients with an ECOG score greater than 2. The $4.9 \%$ ECOG 3 patients included in this study reflects our early experience prior to our full appreciation of the relationship between performance status and postoperative complications.

The completeness of CRS has been shown to be prognostic of survival in younger patients. $^{7,14,19,20}$ Elderly patients with PSD that live longer are those who will have an uncomplicated R0/R1 complete macroscopic cytoreduction. It is important to mention that complete CRS was not prognostic for survival in the presence of postoperative complications, underscoring the substantial impact of a complication in this population. High-volume disease requires aggressive CRS, associated with increased complication rates. Therefore, careful evaluation of preoperative CT and or MRI to determine the volume of 
disease is mandatory. The distribution of disease is also of paramount importance. Disease in the porta hepatis, retroperitoneum, or surrounding major vascular structures not allowing for complete resection should be considered exclusion criteria. In addition, 1 comorbid condition leads to a more than doubling of the odds of having a complication, indicating that comorbidities should be included in the factors guiding preoperative selection along with volume of disease, type of primary, and nutritional status.

The type of primary was also a significant prognosticator of survival. Primaries that do well are low-grade appendiceal cancer, epithelioid mesothelioma, and ovarian cancer. On the contrary, elderly with gastric cancer PSD should not be offered a CRS/HIPEC procedure with the possible exception of patients who present with extremely low volume of disease, notably PCI $<6$ or only positive peritoneal lavage. Colon cancer PSD should also be treated with caution in this population.

Albumin is a well-documented risk factor of operative morbidity and mortality. ${ }^{21}$ In the elderly undergoing CRS/HIPEC, nutritional status as measured by albumin functions as a strong prognostic indicator for survival. Overall, we consider albumin as an indicator of potential postoperative complications. We will postpone surgery until nutritional parameters improve, and we often attempt a 2-week trial of enteral or parenteral nutrition prior to surgery. Complications in the elderly are poorly tolerated because of a lack of physiologic reserves. This is supported by the average hospitalization of 23 days with a complication versus 8 days without. Similarly, the median survival with and without postoperative complications was 13 and 39 months, respectively. In the same context, the type of primary was a less powerful prognostic indicator in the presence of postoperative complication in the specific age group.

Chronologic age has been shown to be prognostic of survival in cohorts of CRS/HIPEC patients that included patients of wide spectrum of ages. ${ }^{20}$ In the narrow range of 70-87 years, age was not significant. In addition, the length of hospitalization was not affected by age. The observed $0 \%$ mortality in the octogenarian group (versus septuagenarians) indicates that chronological age should be taken into consideration in conjunction with the entire clinical picture and not independently.

We recognize that the current paper is limited in that it represents a retrospective review from a single institution. However, it demonstrates accurately the surgical outcomes of CRS/ HIPEC procedures, regardless of primary, on a population that will inevitably continue to grow in the future.

In conclusion, the balance between surgical intervention and quality of life is always of primary importance, but is especially true in the elderly, where maintaining a symptom-free functional status is of paramount significance. The decision to recommend CRS/HIPEC to an elderly patient is complicated by the frequent substantial comorbidities and the poor prognosis of the disease. We do not suggest that CRS/HIPEC should be offered in all patients older than 70 years. On the contrary, it should be offered only to a subset of the elderly, after stringent patient selection based on: type of primary, ability to perform a complete CRS, nutritional status, and performance status. The patient and family should be aware, based on straightforward preoperative discussions, that the procedure is associated with considerable morbidity and mortality and that the quality of life as previously published should be back to baseline within 6 months after the procedure. ${ }^{21,22}$ The procedure should not be attempted without the potential for prolonged survival with acceptable quality of life. Advanced chronological age alone should not be used as a CRS/HIPEC exclusion criterion, but rather placed into consideration along with factors such as type of primary, coexisting comorbidities, and volume of disease. 


\section{References}

1. Etzioni DA, Liu JH, Maggard MA, Ko CY. The aging population and its impact on the surgery workforce. Ann Surg. 2003; 238:170-7. [PubMed: 12894008]

2. Chua TC, Moran BJ, Sugarbaker PH, Levine EA, Glehen O, Gilly FN, et al. Early- and long-term outcome data of patients with pseudomyxoma peritonei from appendiceal origin treated by a strategy of cytoreductive surgery and hyperthermic intraperitoneal chemotherapy. J Clin Oncol. 2012; 30:2449-56. [PubMed: 22614976]

3. Alexander HR, Hanna N, Pingpank JF. Clinical results of cytoreduction and HIPEC for malignant peritoneal mesothelioma. Cancer Treat Res. 2007; 134:343-55. [PubMed: 17633065]

4. Chua TC, Yan TD, Deraco M, Glehen O, Moran BJ, Sugarbaker PH. Multi-institutional experience of diffuse intra-abdominal multicystic peritoneal mesothelioma. Br J Surg. 2011; 98:60-4. [PubMed: 20872843]

5. Elias D, Gilly F, Boutitie F, Quenet F, Bereder JM, Mansvelt B, et al. Peritoneal colorectal carcinomatosis treated with surgery and perioperative intraperitoneal chemotherapy: retrospective analysis of 523 patients from a multicentric French study. J Clin Oncol. 2010; 28:63-8. [PubMed: 19917863]

6. Glehen O, Gilly FN, Boutitie F, Bereder JM, Quenet F, Sideris L, et al. Toward curative treatment of peritoneal carcinomatosis from nonovarian origin by cytoreductive surgery combined with perioperative intraperitoneal chemotherapy: a multi-institutional study of 1,290 patients. Cancer. 2010; 116:5608-18. [PubMed: 20737573]

7. Levine EA, Stewart JH, Russell GB, Geisinger KR, Loggie BL, Shen P. Cytoreductive surgery and intraperitoneal hyperthermic chemotherapy for peritoneal surface malignancy: experience with 501 procedures. J Am Coll Surg. 2007; 204:943-53. [PubMed: 17481516]

8. Verwaal VJ, Bruin S, Boot H, van Slooten G, van Tinteren H. 8-year follow-up of randomized trial: cytoreductions and hyperthermic intraperitoneal chemotherapy versus systemic chemotherapy in patients with peritoneal carcinomatosis of colorectal cancer. Ann Surg Oncol. 2008; 15:2426-32. [PubMed: 18521686]

9. Shen P, Stewart JH, Levine EA. Cytoreductive surgery and hyperthermic intraperitoneal chemotherapy for peritoneal surface malignancy: overview and rationale. Curr Probl Cancer. 2009; 33:125-41. [PubMed: 19647612]

10. Dindo D, Demartines N, Clavien PA. Classification of surgical complications: a new proposal with evaluation in a cohort of 6336 patients and results of a survey. Ann Surg. 2004; 240:205-13. [PubMed: 15273542]

11. Hutchins LF, Unger JM, Crowley JJ, Coltman CA Jr, Albain KS. Underrepresentation of patients 65 years of age or older in cancer-treatment trials. N Engl J Med. 1999; 341:2061-7. [PubMed: 10615079]

12. Polanczyk CA, Marcantonio E, Goldman L, Rohde LE, Orav J, Mangione CM, et al. Impact of age on perioperative complications and length of stay in patients undergoing noncardiac surgery. Ann Intern Med. 2001; 134:637-43. [PubMed: 11304103]

13. Ahmad SA, Kim J, Sussman JJ, Soldano DA, Pennington LJ, James LE, et al. Reduced morbidity following cytoreductive surgery and intraperitoneal hyperthermic chemoperfusion. Ann Surg Oncol. 2004; 11:387-92. [PubMed: 15070598]

14. Shen P, Levine EA, Hall J, Case D, Russell G, Fleming R, et al. Factors predicting survival after intraperitoneal hyperthermic chemotherapy with mitomycin $\mathrm{C}$ after cytoreductive surgery for patients with peritoneal carcinomatosis. Arch Surg. 2003; 138:26-33. [PubMed: 12511145]

15. Kusamura S, Baratti D, Deraco M. Multidimensional analysis of learning curve for cytoreductive surgery and hyperthermic intraperitoneal chemotherapy in peritoneal surface malignancies. Ann Surg. 2011; 255:348-56. [PubMed: 22202584]

16. Extermann M, Overcash J, Lyman GH, Parr J, Balducci L. Comorbidity and functional status are independent in older cancer patients. J Clin Oncol. 1998; 16:1582-7. [PubMed: 9552069]

17. Fried LP, Tangen CM, Walston J, Newman AB, Hirsch C, Gottdiener J, et al. Frailty in older adults: evidence for a phenotype. J Gerontol A Biol Sci Med Sci. 2001; 56:M146-56. [PubMed: 11253156] 
18. da Silva RG, Sugarbaker PH. Analysis of prognostic factors in seventy patients having a complete cytoreduction plus perioperative intraperitoneal chemotherapy for carcinomatosis from colorectal cancer. J Am Coll Surg. 2006; 203:878-86. [PubMed: 17116556]

19. Glehen O, Kwiatkowski F, Sugarbaker PH, Elias D, Levine EA, De Simone M, et al. Cytoreductive surgery combined with perioperative intraperitoneal chemotherapy for the management of peritoneal carcinomatosis from colorectal cancer: a multi-institutional study. J Clin Oncol. 2004; 22:3284-92. [PubMed: 15310771]

20. Gibbs J, Cull W, Henderson W, Daley J, Hur K, Khuri SF. Preoperative serum albumin level as a predictor of operative mortality and morbidity: results from the National VA Surgical Risk Study. Arch Surg. 1999; 134:36-42. [PubMed: 9927128]

21. McQuellon RP, Loggie BW, Lehman AB, Russell GB, Fleming RA, Shen P, et al. Long-term survivorship and quality of life after cytoreductive surgery plus intraperitoneal hyperthermic chemotherapy for peritoneal carcinomatosis. Ann Surg Oncol. 2003; 10:155-62. [PubMed: 12620911]

22. McQuellon RP, Russell GB, Shen P, Stewart JH, Saunders W, Levine EA. Survival and health outcomes after cytoreductive surgery with intraperitoneal hyperthermic chemotherapy for disseminated peritoneal cancer of appendiceal origin. Ann Surg Oncol. 2008; 15:125-33. [PubMed: 18030535] 


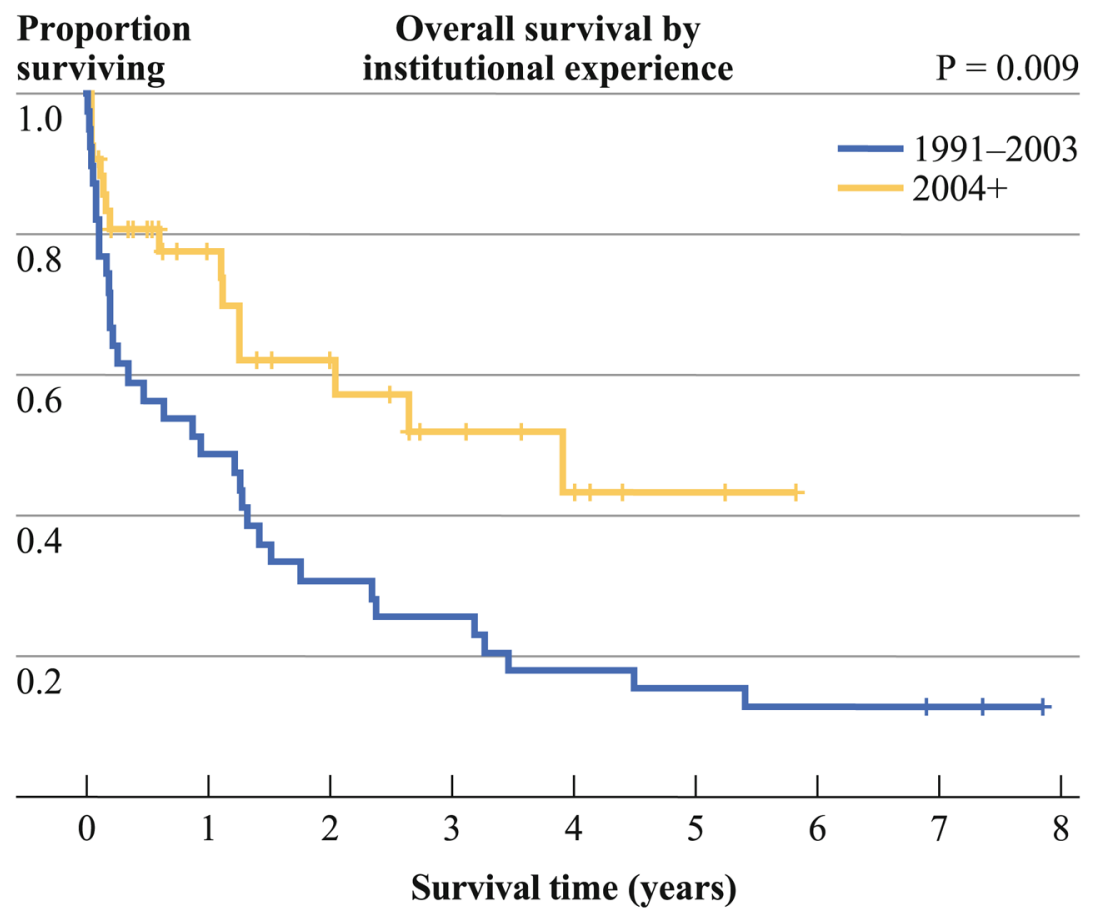

FIG. 1.

Survival curves at the midpoint of institutional surgical experience $(p=0.009)$ 


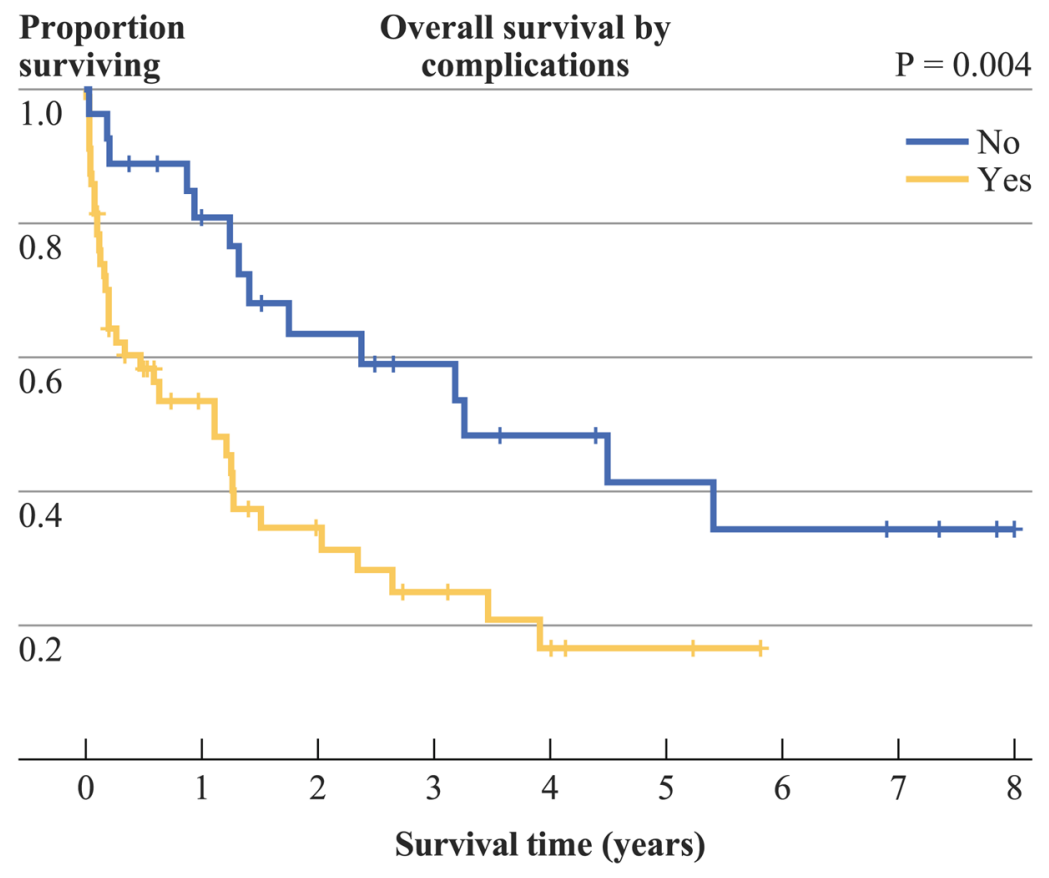

FIG. 2.

Impact of postoperative complications on survival 


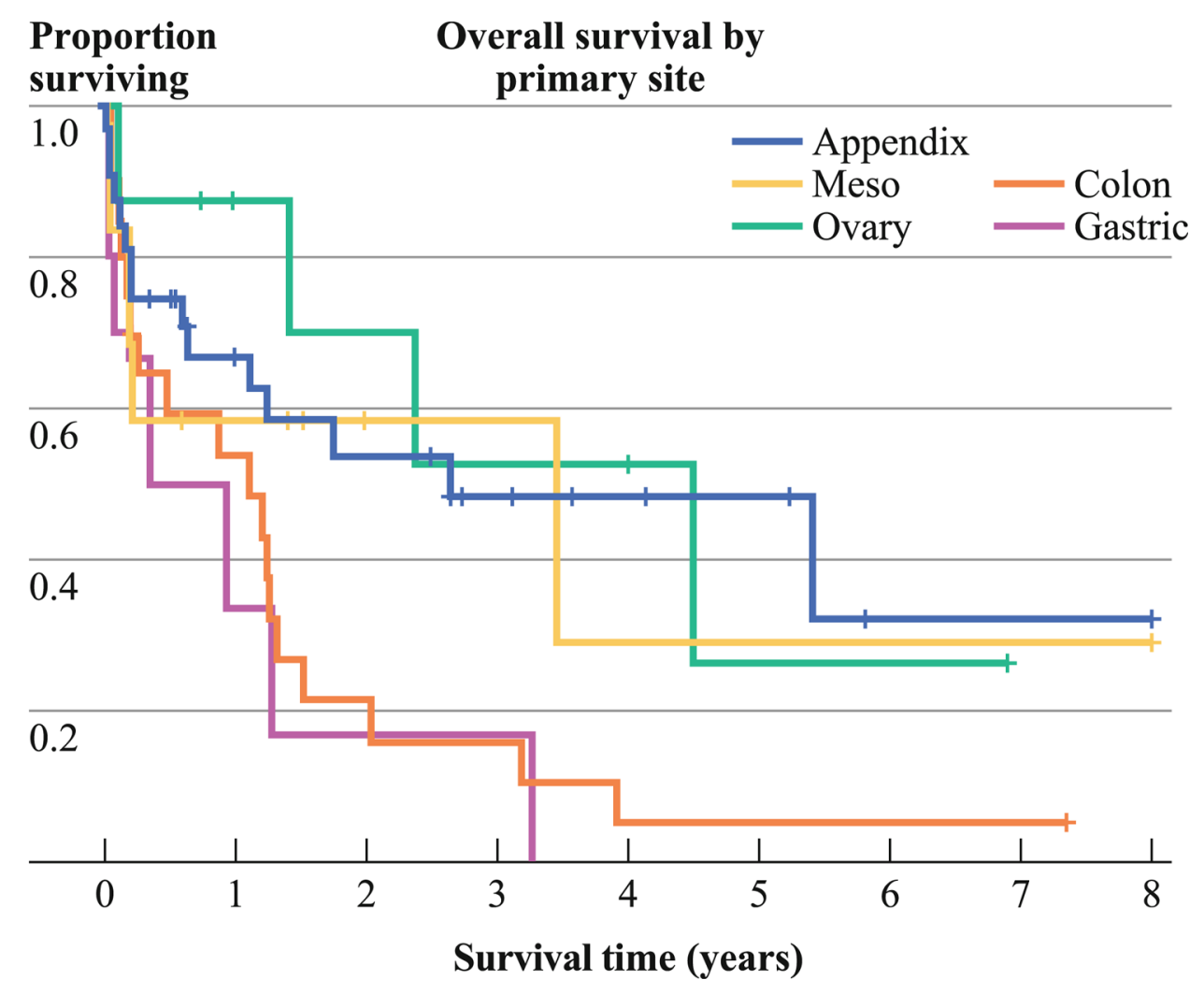

FIG. 3.

Actuarial survival curves comparing survival per primary tumor type $(p=0.03)$ 


\section{TABLE 1}

Clinicopathologic characteristics of 81 elderly patients who underwent CRS/HIPEC for peritoneal carcinomatosis

\begin{tabular}{|c|c|}
\hline Characteristics & $N=81$ \\
\hline Age (years) & $73.4 \pm 3.4$ \\
\hline \multicolumn{2}{|l|}{ Gender } \\
\hline Male & $41(50.6 \%)$ \\
\hline Female & $40(49.4 \%)$ \\
\hline \multicolumn{2}{|l|}{ ECOG status } \\
\hline 0 & $20(24.7 \%)$ \\
\hline 1 & $43(53.1 \%)$ \\
\hline 2 & $14(17.3 \%)$ \\
\hline 3 & $4(4.9 \%)$ \\
\hline $\operatorname{BMI}\left(\mathrm{kg} / \mathrm{m}^{2}\right)$ & $25.9 \pm 4.2$ \\
\hline \multicolumn{2}{|c|}{ Primary tumor $(n=81)$} \\
\hline Appendiceal & $32(39.5 \%)$ \\
\hline Colorectal & $20(24.7 \%)$ \\
\hline Mesothelioma & $10(12.3 \%)$ \\
\hline Ovarian & $8(9.9 \%)$ \\
\hline Gastric & $6(7.4 \%)$ \\
\hline Others & $5(6.2 \%)$ \\
\hline \multicolumn{2}{|c|}{ Resection status after HIPEC } \\
\hline $\mathrm{R} 0-\mathrm{R} 1$ & $36(44.4 \%)$ \\
\hline $\mathrm{R} 2 \mathrm{a}$ & $24(29.6 \%)$ \\
\hline $\mathrm{R} 2 \mathrm{~b}$ & $12(14.8 \%)$ \\
\hline $\mathrm{R} 2 \mathrm{c}$ & $9(11.1 \%)$ \\
\hline \multicolumn{2}{|c|}{ Complication status } \\
\hline No & $27(33.3 \%)$ \\
\hline Yes & $54(66.7 \%)$ \\
\hline Albumin (g/dL) & $3.6 \pm 0.6$ \\
\hline
\end{tabular}


TABLE 2

Predictors of survival

\begin{tabular}{|c|c|c|c|c|}
\hline Univariate predictors of survival & $p$ value & Hazard ratio $(95 \% \mathrm{CI})$ & & \\
\hline Year the procedure was performed & 0.006 & $0.93(0.88-0.98)$ & & \\
\hline Complication & 0.004 & $0.40(0.22-0.77)$ & & \\
\hline Albumin & 0.02 & $0.52(0.35-0.78)$ & & \\
\hline Primary site of origin & 0.03 & & & \\
\hline Appendix vs colon & & $0.43(0.22-0.86)$ & & \\
\hline Appendix vs gastric & & $0.34(0.13-0.89)$ & & \\
\hline Gastric vs ovary & & $3.68(1.03-13.2)$ & & \\
\hline $\mathrm{R}$ resection (R0/R1 vs R2) & 0.10 & $0.62(0.35-1.10)$ & & \\
\hline Gender & 0.77 & NS & & \\
\hline Race & 0.86 & NS & & \\
\hline Age & 0.86 & NS & & \\
\hline ECOG performance status & 0.10 & $1.34(0.95-1.88)$ & & \\
\hline Heart disease & 0.12 & NS & & \\
\hline Nonsmoker & 0.050 & $0.51(0.26-1.01)$ & & \\
\hline Hemoglobin & 0.02 & $0.84(0.72-0.97)$ & & \\
\hline Diabetes & 0.17 & NS & & \\
\hline \multicolumn{3}{|c|}{ Multivariate predictors of survival (complications not included in model) } & $p$ value & Hazard ratio $(95 \% \mathrm{CI})$ \\
\hline \multicolumn{3}{|l|}{ Type of primary } & 0.03 & \\
\hline \multicolumn{3}{|l|}{ Appendix vs colon } & & $0.36(0.17-0.78)$ \\
\hline \multicolumn{3}{|l|}{ Appendix vs gastric } & & $0.25(0.09-0.75)$ \\
\hline \multicolumn{3}{|l|}{ Colon vs ovary } & & $3.68(1.16-11.73)$ \\
\hline \multicolumn{3}{|l|}{ Gastric vs ovary } & & $5.25(1.29-21.33)$ \\
\hline \multicolumn{3}{|l|}{ Albumin } & 0.02 & $0.61(0.40-0.93)$ \\
\hline \multicolumn{3}{|l|}{$\mathrm{R}$ resection } & 0.007 & $0.39(0.20-0.77)$ \\
\hline \multicolumn{3}{|c|}{ Multivariate predictors of survival (complications included in model) } & $p$ value & Hazard ratio $(95 \% \mathrm{CI})$ \\
\hline Year of surgery & & & 0.001 & $0.91(0.87-0.96)$ \\
\hline No complications & & & 0.001 & $0.35(0.18-0.66)$ \\
\hline
\end{tabular}

